\title{
Efference Copy Provides the Eye Position Information Required for Visually Guided Reaching
}

\author{
RICHARD F. LEWIS, ${ }^{1}$ BERTRAND M. GAYMARD, ${ }^{1}$ AND RAFAEL J. TAMARGO ${ }^{2}$ \\ ${ }^{1}$ Department of Neurology and ${ }^{2}$ Department of Neurosurgery, Johns Hopkins University School of Medicine, Baltimore, \\ Maryland 21287
}

Lewis, Richard F., Bertrand M. Gaymard, and Rafael J. Tamargo. Efference copy provides the eye position information required for visually guided reaching. J. Neurophysiol. 80: 16051608,1998 . The contribution of extraocular muscle (EOM) proprioception to the eye position signal used to transform retinotopic visual information to a craniotopic reference frame remains uncertain. In this study we examined the effects of unilateral and bilateral proprioceptive deafferentation of the EOMs on the accuracy of reaching movements directed to visual targets. No significant changes occurred in the mean accuracy (constant error) or variance (variable error) of pointing after unilateral or bilateral deafferentation. We concluded that in normal animals efference copy provides sufficient information about orbital eye position to code space in craniotopic coordinates.

\section{INTRODUCTION}

To transform visual afference from retinotopic to craniotopic coordinates, the brain must combine retinal information with an eye position signal that is derived from extraretinal sources (Skavenski 1990). The eye position signal could be supplied by the feed-forward motor command (efference copy), by proprioceptive afference from the extraocular muscles (EOMs) (Lukas et al. 1994; Ruskell 1978), or by a combination of these two signals. The extraretinal position signal was generally attributed to an efference copy (for review see Carpenter 1988), a view that was supported by eye movement studies that demonstrated no change in saccades after deafferentation of the EOMs (Guthrie et al. 1983). It remains uncertain, however, if saccades require the position of the visual target (VT) to be coded in craniotopic coordinates (Hallet and Lightstone 1976; Mays and Sparks 1980) or if they are coded vectorially in retinotopic coordinates (Goldberg and Bruce 1990; Goldberg and Wurtz 1991; Russo and Bruce 1996).

Recent studies demonstrated that alterations in proprioceptive afference affect the accuracy of reaching movements to VTs (Campos et al. 1986; Gauthier et al. 1990; Lewis et al. 1994; Roll et al. 1991), a motor activity that requires the craniotopic coding of target location. These experiments indicate that EOM proprioception can influence the coding of visual space in certain abnormal circumstances, but the functional significance of the proprioceptive signal remains

The costs of publication of this article were defrayed in part by the payment of page charges. The article must therefore be hereby marked "advertisement" in accordance with 18 U.S.C. Section 1734 solely to indicate this fact. uncertain (Mittelstaedt 1989). It was suggested, for example, that proprioception normally supplies a component of the eye position information used to code visual space (Gauthier et al. 1990), that balanced proprioceptive inputs from the two eyes are required for accurate perception of visual space (Fiorentini et al. 1982; Ventre-Dominey et al. 1996), and that eye position is normally supplied by the efference copy, with proprioception contributing when a mismatch is present between afferent and efferent signals (Lewis et al. 1994).

This study tested the hypothesis that in normal animals efference copy provides sufficient eye position information to transform visual information from retinotopic to craniotopic coordinates. We examined this hypothesis in rhesus monkeys by measuring the effects of unilateral and bilateral proprioceptive deafferentation of the EOMs on the accuracy of reaching movements to VTs.

\section{METHODS}

Experiments were performed on two normal juvenile rhesus monkeys. Surgical procedures were carried out under pentobarbital sodium (30 mg/kg iv) anesthesia, and all animal care procedures complied with the Johns Hopkins Medical School veterinary guidelines. Binocular search coils and a head-restraint plate were surgically implanted. The animals were trained to fixate and point to targets presented on a video monitor in the frontoparallel plane, $24 \mathrm{~cm}$ in front of the animal. Touch position was recorded with a resistive touchscreen on the surface of the monitor and was defined as the initial contact position of the hand on the screen. An elastic band worn on the right (pointing) hand served to minimize the contact area of the fingers on the touchscreen.

The monkey pressed a bar located at waist level with the right hand, a VT appeared straight ahead, and the animal fixed it. After $500 \mathrm{~ms}$ the VT went off and an eccentric, pointing target (PT) appeared along the horizontal axis at eye level. The animal made a saccade to the PT and pointed to it with its right hand.

The animals were trained to point accurately by allowing visual feedback of hand and target position; training PTs ranged in location from left to right $20^{\circ}$ along the horizontal axis but did not include the PT locations used in the testing paradigms. Pointing data were acquired with a visually open-loop testing paradigm. PTs were randomly presented at seven horizontal locations (left $15^{\circ}$ to right $15^{\circ}$, spaced in $5^{\circ}$ intervals ). Testing was carried out in complete darkness; PTs were small $\left(1.4 \mathrm{~mm}^{2}\right)$ and dim (luminance $<0.05 \mathrm{~cd})$ and were extinguished before the hand reached the screen (monitor persistence $<1 \mathrm{~ms}$ ). No visual feedback of pointing accuracy to the target positions used in the testing paradigm was allowed, and hence the animals could not "learn" the positions 

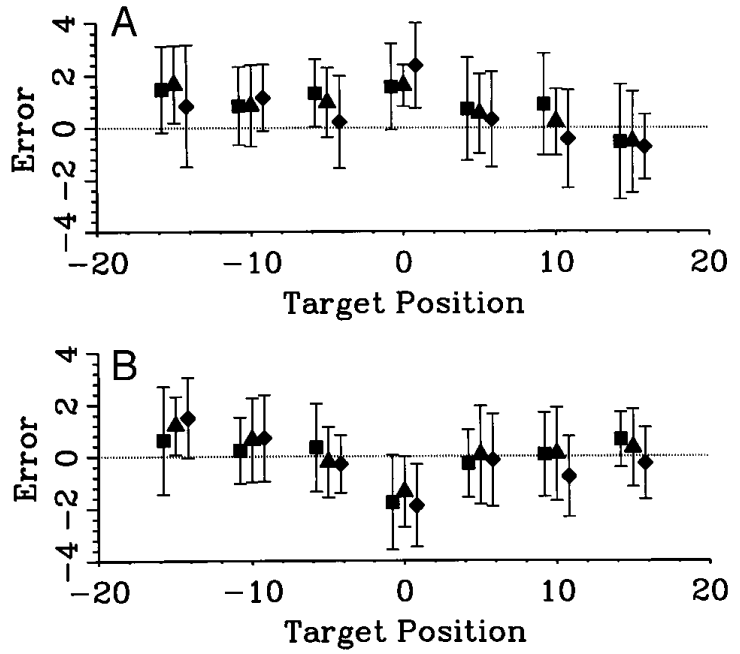

FIG. 1. Horizontal pointing error (touch-target position) as a function of horizontal target location, before deafferentation ( $\boldsymbol{\square})$, after deafferentation of the right eye $(\boldsymbol{\Delta})$, and after bilateral deafferentation $(\bullet)$. Each icon represents the average of 20-30 pointing values for each target position, and error bars indicate SD. Icons are offset horizontally for clarity, and all values are in degrees. $A$ : monkey $S$, binocular viewing; $B$ : monkey $C$, right eye viewing.

of the testing targets in extrapersonal space. The absence of visual feedback in the testing condition was confirmed by studying pointing while the animals viewed through a wedge prism (Held and Freedman 1963). No compensatory corrections to the prism-induced displacement of the PT occurred, nor did adaptive modification of pointing.

After the animals were trained, they were tested 3 days/wk for 3 wk. Data were acquired in binocular (BEV) and monocular right eye viewing (REV) conditions. The EOMs of the right eye were then proprioceptively deafferented by sectioning the ophthalmic division of the trigeminal nerve (Porter et al. 1983) immediately distal to the Gasserian ganglion, and the animals were tested for $1 \mathrm{wk}$. The ophthalmic division on the left was then sectioned, and pointing was tested for $3 \mathrm{wk}$. The ophthalmic division was identified anatomically and physiologically (electrical stimulation evoked a blink but no eye movement) at surgery, and the corneal reflex was absent throughout the postoperative period.

Data were quantified as horizontal pointing error (touch-target position) and analyzed as a function of target position and proprioceptive state with two-way analysis of variance (ANOVA). Constant error before and after deafferentation was compared statistically with ANOVA and Student's $t$-test, and variable error was compared with a $F$ test.

\section{RESULTS}

Before deafferentation, the monkeys pointed accurately to VTs in both viewing conditions (Fig. 1, Table 1). For each target location, constant error was typically between 0.5 and $1.5^{\circ}$ and variable error (SD) ranged from 1.0 to $2.0^{\circ}$ (Fig. 1). No significant change occurred in constant pointing error after unilateral or bilateral proprioceptive deafferentation in either monkey (Fig. 1, Table 1). Changes did not occur acutely after deafferentation or gradually in the subsequent weeks of investigation (Fig. 2). Although constant error varied significantly with target location before and after deafferentation $(P<0.01$, ANOVA, for both monkeys and viewing conditions), it was not affected by deafferentation (AN-
TABLE 1. Effect of deafferentation on pointing

\begin{tabular}{rcccr}
\hline \hline & Pre-deaff & RE-deaff & BE-deaff & $P$ Value \\
\hline \multicolumn{5}{c}{ Monkey $S$} \\
BEV: Mean error: & $0.91(196)$ & $0.77(121)$ & $0.53(199)$ & 0.12 \\
SD: & 1.86 & 1.60 & 1.97 & $>0.05$ \\
REV: Mean error: & $-0.76(127)$ & $-0.59(127)$ & $-0.90(182)$ & 0.16 \\
SD: & 1.37 & 1.18 & 1.54 & $>0.05$ \\
& \multicolumn{5}{c}{ Monkey C } \\
BEV: Mean error: & $1.00(114)$ & $0.83(110)$ & $0.82(171)$ & 0.63 \\
SD: & 1.67 & 2.10 & 1.44 & $>0.05$ \\
REV: Mean error: & $0.03(106)$ & $0.11(125)$ & $-0.10(105)$ & 0.62 \\
SD: & 1.71 & 1.66 & 1.85 & $>0.05$ \\
\hline
\end{tabular}

Values are the mean pointing error (combining the horizontal target locations), number of observations (in parentheses), and standard deviation (SD), for each animal in the two viewing conditions before proprioceptive deafferentation ("Pre-deaff") and after deafferentation of the right eye ('RE-deaff') and both eyes ("BE-deaff') $P$ values are determined with an analysis of variance (means) and $F$ test (comparing the $\mathrm{SD}^{2}$ in the Predeaff and BE-deaff states).

OVA; $P=0.19$, monkey $S, \mathrm{BEV} ; P=0.74$, monkey $S$, $\mathrm{REV} ; P=0.61$, monkey $C, \mathrm{BEV} ; P=0.45$ monkey $C$, REV). Mean constant error averaged over the seven target locations (Table 1) was also unaffected by deafferentation ( $P>0.12$ for both monkeys and viewing conditions ).

Variable error of pointing responses was not significantly affected by bilateral proprioceptive deafferentation (Table $1, F$ test $>0.05$ for each animal and viewing condition). Variable error increased (nonsignificantly) after bilateral deafferentation in three of the four monkey-testing conditions and decreased in the other condition (Table 1).

\section{I S C U S S I O N}

The results demonstrate that the accuracy and variability of pointing movements to VTs are unaffected by unilateral and bilateral proprioceptive deafferentation of the EOMs. This finding indicates that the efference copy provides sufficient information to the brain to accurately encode orbital eye position in normal animals. It does not, however, exclude the possibility that eye position is normally coded by a combination of proprioceptive and efferent signals (Gauthier et

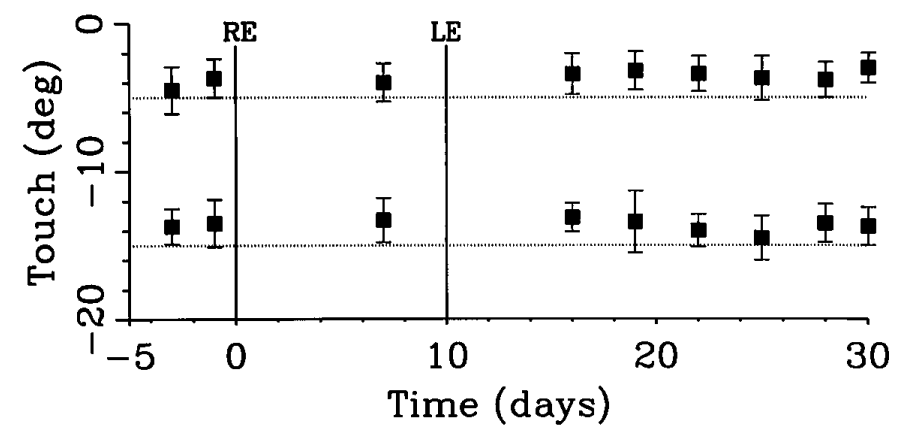

FIG. 2. Mean pointing location (in deg, monkey $S$, BEV condition) as a function of time, for two target positions (left $5^{\circ}$, left $15^{\circ}$ ), before proprioceptive deafferentation, after deafferentation of the right eye (RE), and after subsequent deafferentation of the left eye (LE). Error bars indicate SD. 
al. 1990), as adaptive mechanisms could potentially compensate for the proprioceptive signal after deafferentation. Another possibility is that proprioception and efference copy normally provide redundant sources of eye position information. In this situation, deafferentation would not affect constant pointing error. It might be expected to produce an increase in variable error, however, as there is behavioral (Desmurget et al. 1995) and neuronal (Meredith and Stein 1986) evidence that central representations of physical parameters may be sharpened by congruent inputs. Finally, given the role of proprioception in the development of depth perception (Trotter et al. 1993) and the control of ocular alignment (Lewis et al. 1994), deafferentation could potentially affect the amplitude of reaching movements in depth rather than the direction of movement. This effect could produce inaccuracy of limb movements in three-dimensional space, which was not studied in this experimental paradigm.

Two prior experiments investigated the effect of unilateral ocular deafferentation on visually guided motor activity and reported that the direction of jumping (Fiorentini et al. 1982) and reaching (Ventre-Dominey et al. 1996) movements shifted toward the deafferented eye. These authors suggested that the shifts resulted from an imbalance between the afferent signals of the two eyes and hypothesized that accurate coding of visual space depends on balanced proprioceptive afference from both eyes. Our results, however, demonstrate no change in pointing accuracy after unilateral ocular deafferentation. Although there are multiple methodological differences between studies, Ventre-Dominey et al. (1996) investigated patients with trigeminal neuralgia, a condition that could be associated with aberrant proprioceptive afference before deafferentation. Furthermore, the ophthalmic division was not directly visualized in their study, so it may have been damaged but not completely destroyed by the coagulation procedure, thereby altering the afferent signal without eliminating it. The persistence of normal pointing after unilateral deafferentation in our study implies that balanced afferent inputs from the two eyes are not required for accurate spatial localization and suggests that the change in localization that occurs when afference from one eye is modified (i.e., Gauthier et al. 1990) is not caused by an imbalance between the afferent signals of the two eyes.

A basic assertion underlying our study (and other investigations of ocular proprioception) is that sectioning the ophthalmic division of the trigeminal nerve produces a complete (or nearly complete) proprioceptive deafferentation of the EOMs. This assertion is based primarily on the anatomic studies of Porter et al. (1983), who demonstrated that injection of retrograde tracer into the EOMs resulted in cellular labeling in the ipsilateral trigeminal ganglion but no labeling in the trigeminal mesencephalic nucleus, the potential central site of afferent cell bodies. Ganglionic labeling was blocked when the ophthalmic division of the trigeminal nerve was sectioned before tracer injection. These findings were questioned by Gentle and Ruskell (1997), who used degeneration methodology and suggested that proprioceptive fibers may travel to the brain stem in the ocular motor nerves, not the trigeminal. Tracer studies confirm, however, that the cell bodies of the afferent nerves that innervate the EOMs are located in the trigeminal ganglion (Billig et al. 1997; Porter
1986). Section of the ophthalmic division immediately distal to the ganglion would therefore eliminate most or all of the afferent innervation of the EOMs, even if some sensory fibers cross back to the motor nerves proximal to the ganglion.

We thank D. S. Zee and D. A. Robinson for reviewing the manuscript and also C. Bridges, D. Roberts, and A. Lasker.

This work was supported by National Institute of Neurological Disorders and Stroke Grant NS-01656 to R. F. Lewis, by the Institute National de la Sante et de la Recherche Medicale to B. M. Gaymard, and by Grant RG58/ 92B from the Human Frontier Science Program.

Address for reprint requests: R. F. Lewis, Pathology 2-210, 600 N. Wolfe St., Baltimore, MD 21287-6121.

Received 1 April 1998; accepted in final form 26 May 1998.

\section{REFERENCES}

Billig, I., Buisseret, C., AND Buisseret, P. Identification of nerve endings in cat extraocular muscles. Anat. Rec. 248: 566-575, 1997.

Campos, E. C., Chiesi, C., And Bolzani, R. Abnormal spatial localization in patients with herpes zoster ophthalmicus: evidence for the presence of proprioceptive information. Arch. Ophthalmol. 104: 1176-1177, 1986.

Carpenter, R.H.S. Movements of the Eyes. London: Pion, 1988, p. $293-$ 312.

Desmurget, M., Rossetti, Y., Prablanc, C., Stelmach, G., And JEANNEROD, M. Representation of hand position prior to movement and motor variability. Can. J. Physiol. Pharm. 73: 262-272, 1995.

FIORENTINI, A., BERARDI, N., AND MAFFEI, L. Role of extraocular proprioception in the orienting behavior of cats. Exp. Brain Res. 48: 113-120, 1982.

Gauthier, G. M., Nommay, D., And Vercher, J.-L. Ocular muscle proprioception and visual localization of targets in man. Brain 113: $1857-$ $1871,1990$.

Gentle, A. And Ruskell, G. Pathway of the primary afferent nerve fibres serving proprioception in monkey extraocular muscles. Ophthalmic Physiol. Opt. 17: 225-231, 1997.

Goldberg, M. E. And Bruce, C. J. Primate frontal eye fields. III. Maintenance of a spatially accurate saccade signal. J. Neurophysiol. 64: 489508, 1990.

GoldberG, M. E. AND Wurtz, R. H. Extraretinal influences on the visual control of eye movement. In: Motor Control: Concepts and Issues, edited by D. R. Humphrey and H.-J. Freund. Chichester, UK: Wiley, 1991, p. $163-179$.

Guthrie, B. L., Porter, J. D., And Sparks, D. L. Corollary discharge provides accurate eye position information to the oculomotor system. Science 221: 1193-1195, 1983.

Hallet, P. E. And Lightstone, A. D. Saccadic eye movements towards stimuli triggered by prior saccades. Vision Res. 16: 99-106, 1976.

Held, R. AND Freedman, S. J. Plasticity in human sensorimotor control. Science 142: 455-462, 1963.

LEWIS, R. F. AND ZEE, D. S. Abnormal spatial localization with trigeminaloculomotor synkinesis: evidence for a proprioceptive effect. Brain 116: 1105-1118, 1993.

Lewis, R. F., Zee, D. S., Gaymard, R. F., And Guthrie, B. L. Extraocular muscle proprioception functions in the control of ocular alignment and eye movement conjugacy. J. Neurophysiol. 72: 1028-1031, 1994.

Lukas, J. R., Aigner, M., Blumer, R., Heinzl, H., and May, R. Number and distribution of neuromuscular spindles in human extraocular muscles. Invest. Ophthalmol. Vis. Sci. 35: 4317-4327, 1994.

MAYS, L. E. AND SPARKS, D. L. Saccades are spatially, not retinocentrically, coded. Science 208: 1163-1165, 1980.

Meredith, M. A. AND Stein, B. E. Visual, auditory, and somatosensory convergence on cells in superior colliculus results in multisensory integration. J. Neurophysiol. 56: 640-653, 1986.

MitTELstAedT, H. Basic solutions to the problem of head-centric visual localization. In: The Perception and Control of Self Motion, edited by R. Warren and A. H. Wertheim. Hillsdale, NJ: Erlbaum, 1989, p. 267-287.

PORTER, J. D. Brainstem terminations of extraocular muscle primary afferent neurons in the monkey. J. Comp. Neurol. 247: 133-143, 1986.

Porter, J. D., Guthrie, B. L., AND Sparks, D. L. Innervation of monkey extraocular muscles: localization of sensory and motor neurons by retro- 
grade transport of horseradish peroxidase. J. Comp. Neurol. 218: 208219, 1983

Roll, R., Velay, J. L., AND Roll, J. P. Eye and neck proprioceptive messages contribute to the spatial coding of retinal input in visually oriented activities. Exp. Brain Res. 85: 423-431, 1991.

RuSKELL, G. L. The fine structure of innervated myotendinous cylinders in extraocular muscles of rhesus monkeys. J. Neurocytol. 7: 693-708, 1978.

Russo, G. S. AND BRuCE, C. J. Neurons in the supplementary eye field of rhesus monkeys code visual targets and saccadic eye movements in an oculocentric coordinate system. J. Neurophysiol. 76: 825-848, 1996.
SKAVENSKI, A. A. Eye movement and visual localization of objects in space. In: Eye Movements and Their Role in Visual and Cognitive Processes, edited by E. Kowler. Amsterdam: Elsevier, 1990, p. 263-287.

Trotter, Y., Celebrini, S., Beaux, J.-C., Grandjean, B., and Imbert, M. Long-term dysfunctions of neural stereoscopic mechanisms after unilateral extraocular muscle proprioceptive deafferentation. J. Neurophysiol. 69: 1513-1529, 1993.

Ventre-Dominey, J., Dominey, P. F., AND Sindou, M. Extraocular proprioception is required for spatial localization in man. NeuroReport 7: 1531$1535,1996$. 